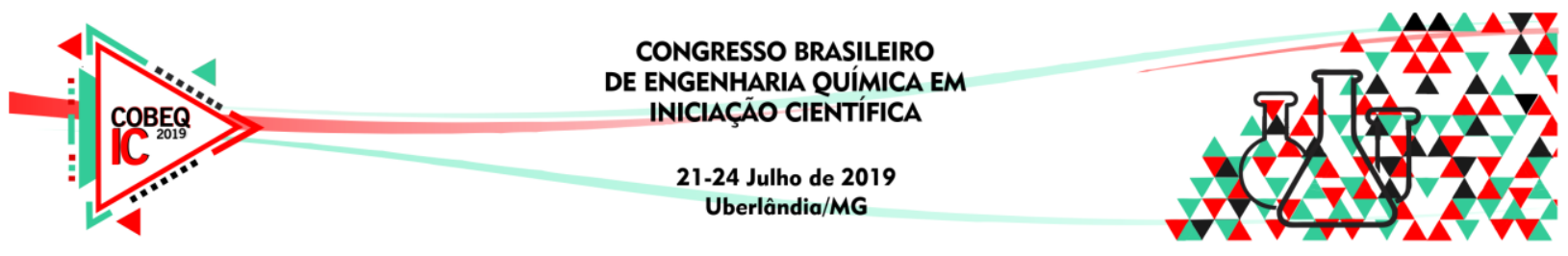

\title{
CINÉTICA E EQUILÍBRIO DA ADSORÇÃO DO CORANTE AZUL DE METILENO UTILIZANDO BAGAÇO DE CANA E FIBRA DE COCO COMO ADSORVENTES
}

\author{
B. VIGANÔ ${ }^{1}$, I. A. GONTIJO ${ }^{1}$ e S. C. DANTAS ${ }^{1}$ \\ ${ }^{1}$ Universidade Federal do Triângulo Mineiro, Departamento de Engenharia Química \\ E-mail para contato: sandra.dantas@uftm.edu.br
}

\begin{abstract}
RESUMO - O azul de metileno é um corante empregado em larga escala industrialmente, em especial na indústria têxtil. Com o intuito de minimizar o impacto ambiental gerado pelo seu descarte, este trabalho teve como objetivo estudar a cinética e o equilíbrio de adsorção deste corante utilizando como materiais adsorventes fibra de coco e bagaço de cana de açúcar. Para cada um dos adsorventes, foram realizados dois tratamentos, sendo o primeiro com $\mathrm{NaOH}$ 0,1 $\mathrm{M}$ e o segundo com $\mathrm{HCl}$ 0,5 $\mathrm{M}$, totalizando 6 tipos de adsorventes, visto que eles também foram utilizados in natura. Todos os testes foram realizados utilizando $300 \mathrm{~mL}$ de solução (nas concentrações de 5, 30, 50, 100 e $300 \mathrm{mg} / \mathrm{L}$ ) para $3 \mathrm{~g}$ de fibra. Comparando os resultados obtidos pelas fibras com tratamento, com as que não sofreram nenhum tipo de alteração química (in natura), observouse uma melhora de até $62 \%$ na eficiência de adsorção para o bagaço de cana tratado, e de até $28 \%$ para a fibra de coco tratada. Além disso, para todos os testes, os dados cinéticos foram melhores ajustados para o modelo de pseudo-segundaordem, enquanto os dados de equilíbrio se ajustaram para o modelo de Freundlich.
\end{abstract}

\section{INTRODUÇÃO}

Uma das principais formas de contaminação da água é através do lançamento indireto de corantes nos corpos d'água, que são utilizados em diversos processos industriais, sendo esta, uma grande fonte de poluição da água. A partir deste contexto ambiental, surge a necessidade do desenvolvimento de tecnologias para tratar de forma adequada este tipo de efluente. Um dos processos para a remoção do corante é a adsorção (Lavich, 2003).

O carvão ativado é um dos adsorventes mais usados no tratamento de efluentes através da adsorção, segundo Guilarduci et al. (2006), mas é notória sua inviabilidade devido ao alto valor agregado. Com isso, surgem estudos para minimizar o custo deste processo, utilizando subprodutos industriais ou resíduos orgânicos. Dentre as fibras mais comuns encontradas no Brasil, estão as fibras de coco, da bananeira, das palhas de arroz e trigo, e do bagaço de canade-açúcar. Para melhorar a atividade destes bioadsorventes, é comum o tratamento químico destes materiais com soluções de ácidos e/ou de bases (Albinante et al., 2013).

Com isso, este trabalho teve como objetivo realizar o estudo da cinética e equilíbrio da adsorção do corante azul de metileno, utilizando como adsorventes o bagaço de cana de 


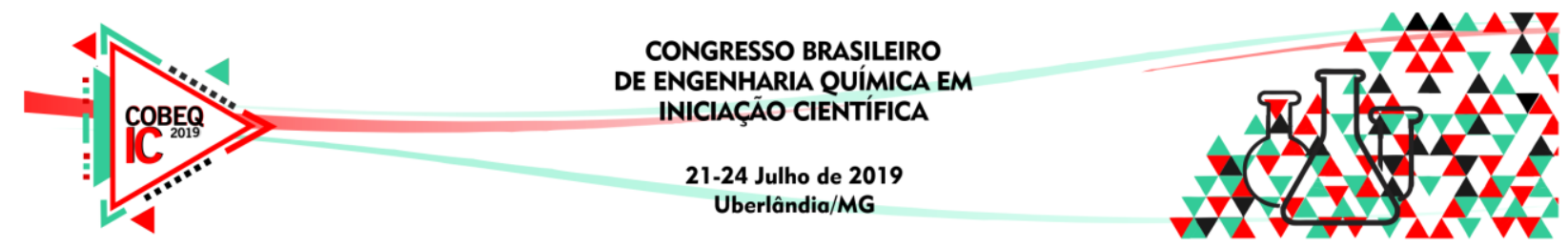

açúcar e fibras de coco, sendo que ambos foram tratados quimicamente com ácido e com base.

\section{PARTE EXPERIMENTAL}

\subsection{Preparo dos Materiais Adsorventes}

Com o intuito de padronizar e otimizar o estudo, após a coleta e limpeza, as fibras de coco e de bagaço de cana foram lavadas e colocadas na estufa durante $24 \mathrm{~h}$ a $70^{\circ} \mathrm{C}$. Em seguida, houve a moagem da biomassa, utilizando um moinho de facas. Por fim, cada um dos materiais foi peneirado, utilizando nos testes apenas a biomassa retida entre 16\# e 60\#, em ambos os casos.

Após o peneiramento, as fibras passaram por dois tratamentos distintos, sendo um a ativação básica, onde se colocou $1 / 3$ do material previamente seco e moído em contato com $\mathrm{NaOH}$ 0,1 M durante 18 h (Furmanski et al., 2012), sem agitação. Paralelamente, outro 1/3 do material passou pela ativação ácida, na qual a biomassa foi posta em contato com $\mathrm{HCl} 0,5 \mathrm{M}$, baseada na concentração utilizada por Silva e Oliveira (2012), nas mesmas condições. O 1/3 restante do bagaço de cana e da fibra de coco foram mantidas in natura. Posteriormente, as biomassas tratadas foram lavadas, e postas na estufa durante $24 \mathrm{~h} \mathrm{a} 60^{\circ} \mathrm{C}$. Dessa forma, foram avaliadas como adsorventes duas biomassas, cada uma com três condições diferentes: Bagaço de cana in natura (BCI), com ativação ácida (BCA), com ativação básica (BCB) e fibra de coco in natura (FBI), com ativação ácida (FCA) e com ativação básica (FCB).

\subsection{Cinética e Isotermas de Adsorção}

Para realização dos testes, foram utilizadas cinco concentrações distintas de solução de azul de metileno, sendo elas de $5 \mathrm{mg} / \mathrm{L}, 30 \mathrm{mg} / \mathrm{L}, 50 \mathrm{mg} / \mathrm{L}, 100 \mathrm{mg} / \mathrm{L}$ e $300 \mathrm{mg} / \mathrm{L}$. Em cada teste, foram retiradas alíquotas em diferentes intervalos de tempo. Considerou-se a concentração de equilíbrio a última medida de cada teste, onde já não havia mais variação da concentração da solução, que foi determinada com auxílio do espectrofotômetro, ajustado em $664 \mathrm{~nm}$, assim como no estudo de Jorge et al. (2015).

Os modelos cinéticos utilizados foram os de pseudo-primeira ordem, de pseudo-segunda ordem e de difusão intrapartícula (Bertolini e Fungaro, 2011) e estão apresentados na Tabela 1. Para determinação dos parâmetros de cada um dos modelos foi feito o ajuste linear.

Tabela 1 - Modelos cinéticos de adsorção.

\begin{tabular}{|c|c|c|}
\hline Pseudo-primeira-ordem & Pseudo-segunda-ordem & Difusão intrapartícula \\
\hline $\log \left(q_{e}-q\right)=\log q_{e}-\frac{k_{1}}{2,303} t$ & $\frac{\mathrm{t}}{\mathrm{q}}=\frac{1}{\mathrm{k}_{2} \mathrm{qe}^{2}}+\frac{1}{\mathrm{qe}} \mathrm{t}$ & $\mathrm{qt}=\mathrm{kdif.}^{1 / 2}+\mathrm{C}$ \\
\hline
\end{tabular}

O parâmetro k2 é usado para determinar a velocidade de adsorção: $\mathrm{h}=\mathrm{k} 2 \mathrm{q}^{2}$ 


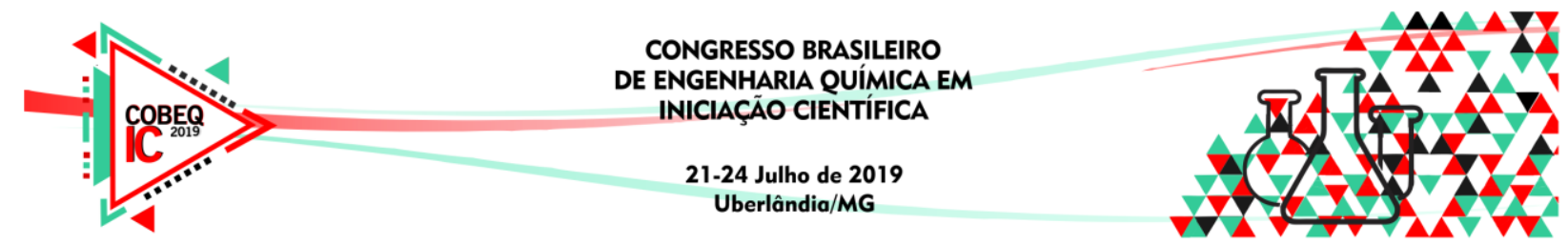

A eficiência de adsorção (R) e a quantidade de corante adsorvido (q) foram calculadas a partir da Equação 2 e da Equação 3, respectivamente.

$$
\begin{aligned}
& R=100 \frac{\left(C_{0}-C_{f}\right)}{C_{0}} \\
& q=V \frac{\left(C_{0}-C_{f}\right)}{M}
\end{aligned}
$$

Já para o estudo de equilíbrio de adsorção, utilizando as concentrações de equilíbrio obtidas no final de cada experimento $\left(\mathrm{q}_{\mathrm{e}}\right)$, foram comparados cinco modelos de isotermas de adsorção, de acordo com Colombo (2013), expostos na Tabela 2. Estes modelos passaram por um ajuste não linear.

\begin{tabular}{|c|c|c|c|c|}
\hline $\begin{array}{l}\text { Isoterma de } \\
\text { Langmuir }\end{array}$ & $\begin{array}{l}\text { Isoterma de } \\
\text { Freundlich }\end{array}$ & Isoterma de Tóth & $\begin{array}{c}\text { Isoterma de Radke- } \\
\text { Praunsnitz }\end{array}$ & Isoterma de Sips \\
\hline $\mathrm{qe}_{\mathrm{e}}=\frac{\mathrm{q}_{\mathrm{máx}} \mathrm{KLCe}_{\mathrm{L}}}{1+\mathrm{KLCe}_{\mathrm{L}}}$ & $\mathrm{qe}=\mathrm{KFCe}^{1 / \mathrm{nF}}$ & $\mathrm{q}_{\mathrm{e}}=\frac{\mathrm{qmáx}^{\mathrm{K}} \mathrm{KTCe}_{\mathrm{T}}}{\left(1+\mathrm{KTC}_{\mathrm{TT}}\right)^{1 / \mathrm{nT}}}$ & $\mathrm{qe}_{\mathrm{e}}=\frac{\mathrm{q}_{\mathrm{máx} K R P C e}}{\left(1+\mathrm{KRPCe}_{\mathrm{e}}\right)^{\mathrm{nRP}}}$ & $\mathrm{qe}_{\mathrm{e}}=\frac{\mathrm{qmáx}\left(\mathrm{KsC} \mathrm{Ce}^{\mathrm{nS}}\right.}{1+\left(\mathrm{KsCe}_{\mathrm{n}}\right)^{\mathrm{nS}}}$ \\
\hline
\end{tabular}

Tabela 2 - Modelos de isotermas de adsorção.

\section{RESULTADOS}

Para determinar as concentrações das amostras coletadas durante os testes, foi utilizada uma curva de calibração, que relaciona a leitura da absorbância (x) com sua respectiva concentração (y). A curva foi construída utilizando concentrações conhecidas de solução e a equação da reta resultante igual a $\mathrm{y}=0,0742 \mathrm{x}+0,0208$, cujo coeficiente de determinação $\left(\mathrm{r}^{2}\right)$ foi de 0,9825 .

\subsection{Cinética de Adsorção}

Para cada um dos trinta testes, os dados experimentais foram ajustados para determinação da cinética de adsorção. Dessa forma, obteve-se os parâmetros cinéticos de cada modelo para ambas as fibras, de coco e de bagaço de cana, de modo que, em todos os experimentos realizados o modelo de melhor ajuste, de acordo com a análise do valor de $\mathrm{r}^{2}$, foi o de pseudo-segunda-ordem. Sendo assim, os parâmetros cinéticos do bagaço de cana estão expostos na Tabela 3, enquanto da fibra de coco encontram-se na Tabela 4.

Tabela 3 - Parâmetros cinéticos de pseudo-segunda-ordem do bagaço de cana

\begin{tabular}{|c|c|c|c|c|c|c|c|c|c|}
\hline & \multicolumn{3}{|c|}{ In natura } & \multicolumn{3}{c|}{ Ativação ácida } & \multicolumn{3}{c|}{ Ativação básica } \\
\hline \hline Conc. & $\mathrm{k}_{2}$ & $\mathrm{~h}$ & $\mathrm{r}^{2}$ & $\mathrm{k}_{2}$ & $\mathrm{~h}$ & $\mathrm{r}^{2}$ & $\mathrm{k}_{2}$ & $\mathrm{~h}$ & $\mathrm{r}^{2}$ \\
\hline 5 & 2,4836 & 0,4088 & 0,9999 & 2,2568 & 0,4517 & 0,9999 & 2,1772 & 0,4603 & 1,0000 \\
\hline 30 & 0,4288 & 2,4056 & 0,9999 & 0,4266 & 2,5820 & 0,9982 & 0,3507 & 2,8637 & 1,0000 \\
\hline 50 & 0,2607 & 3,9231 & 0,9999 & 0,2528 & 4,1754 & 0,9997 & 0,2155 & 4,6707 & 1,0000 \\
\hline 100 & 0,1711 & 6,2383 & 0,9989 & 0,1506 & 7,6805 & 0,9960 & 0,1052 & 9,5602 & 1,0000 \\
\hline 300 & 0,1028 & 10,6952 & 0,9976 & 0,0753 & 15,6006 & 0,9931 & 0,0367 & 27,8552 & 0,9998 \\
\hline
\end{tabular}




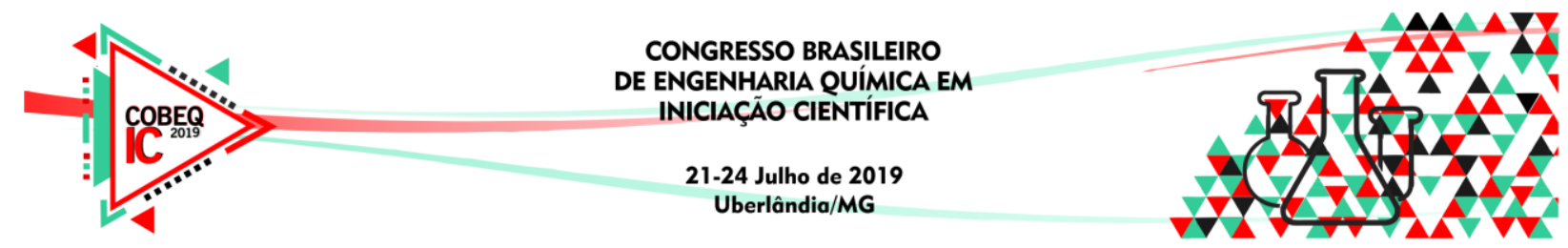

Tabela 4 - Parâmetros cinéticos de pseudo-segunda-ordem da fibra de coco

\begin{tabular}{|c|c|c|c|c|c|c|c|c|c|}
\hline & \multicolumn{3}{|c|}{ In natura } & \multicolumn{3}{c|}{ Ativação ácida } & \multicolumn{3}{c|}{ Ativação básica } \\
\hline \hline Conc. & $\mathrm{k}_{2}$ & $\mathrm{~h}$ & $\mathrm{r}^{2}$ & $\mathrm{k}_{2}$ & $\mathrm{~h}$ & $\mathrm{r}^{2}$ & $\mathrm{k}_{2}$ & $\mathrm{~h}$ & $\mathrm{r}^{2}$ \\
\hline 5 & 1,9710 & 0,5252 & 0,9997 & 1,4087 & 0,7297 & 0,9997 & 1,5440 & 0,6679 & 0,9997 \\
\hline 30 & 0,4551 & 2,2297 & 0,9996 & 0,3849 & 2,7337 & 0,9994 & 0,3803 & 2,7226 & 0,9998 \\
\hline 50 & 0,3173 & 3,9761 & 0,9997 & 0,2573 & 4,3898 & 0,9901 & 0,2377 & 4,3066 & 0,9999 \\
\hline 100 & 0,1335 & 7,8003 & 0,9999 & 0,1153 & 9,2764 & 0,9993 & 0,1110 & 9,3633 & 0,9998 \\
\hline 300 & 0,0430 & 24,4499 & 0,9996 & 0,0440 & 24,8139 & 0,9987 & 0,0385 & 27,1003 & 0,9997 \\
\hline
\end{tabular}

A partir dos dados obtidos experimentalmente, foi possível observar que a velocidade inicial de adsorção (h) aumenta proporcionalmente à concentração da solução. Isso se deve ao fato de uma solução mais concentrada apresentar maior quantidade de moléculas de corante disponíveis para serem aderidas à superfície do sólido. Além disso, notou-se que os tratamentos dos materiais adsorventes apresentaram resultados positivos, assim como no estudo realizado por Furmanski et al. (2012), uma vez que para ambas as fibras a velocidade inicial de adsorção foi maior no material previamente tratado do que naqueles in natura.

Através da eficiência de adsorção, Tabela 5, observou-se que o bagaço de cana tratado apresentou uma melhora mais acentuada do que a fibra de coco sob as mesmas condições. Isso possivelmente ocorreu por causa das diferentes composições das duas fibras utilizadas, de modo que quanto maior for a porcentagem de celulose em sua composição, maior será a sua capacidade de adsorção, visto que a possibilidade de interação entre os grupos funcionais da celulose e do azul de metileno são maiores (Furmanski et al, 2012).

Tabela 5 - Eficiência de adsorção (R)

\begin{tabular}{|c|c|c|c|c|c|}
\hline & $5 \mathrm{mg} / \mathrm{L}$ & $30 \mathrm{mg} / \mathrm{L}$ & $50 \mathrm{mg} / \mathrm{L}$ & $100 \mathrm{mg} / \mathrm{L}$ & $300 \mathrm{mg} / \mathrm{L}$ \\
\hline \hline BCI & 88,2181 & 81,4516 & 82,1331 & 62,0760 & 36,2029 \\
\hline BCA & 97,3036 & 84,6033 & 86,0420 & 73,4067 & 51,0675 \\
\hline BCB & 100,0000 & 98,2759 & 98,5677 & 97,9883 & 97,7799 \\
\hline FCI & 68,6134 & 76,1216 & 75,5465 & 80,1495 & 84,6036 \\
\hline FCA & 95,6646 & 91,6481 & 88,1443 & 92,8141 & 84,2688 \\
\hline FCB & 87,4239 & 92,0189 & 90,8479 & 95,0597 & 94,1862 \\
\hline
\end{tabular}

\subsection{Isotermas de Adsorção}

Associando os parâmetros de concentração da solução e quantidade de soluto adsorvida, ambos no equilíbrio, foram obtidas as isotermas de adsorção, seguindo os modelos apresentados anteriormente e realizado o ajuste não linear. Os parâmetros resultantes do ajuste não linear podem ser observados nas Tabelas 6 e 7.

A partir da análise do $\mathrm{R}^{2}$ dos ajustes realizados para o bagaço de cana in natura, o melhor modelo seria o de Radke-Praunsnitz. Porém, seus parâmetros apresentaram desvios padrão elevados, indicando que houve uma grande dispersão dos dados. Portanto, observando juntamente ao desvio padrão, notou-se que o melhor modelo neste caso foi o de Freundlich, já que os parâmetros apresentaram uma variação razoável. 


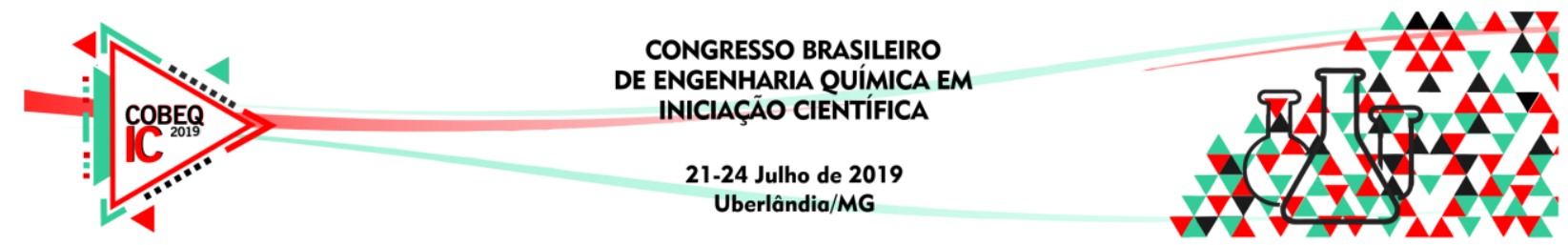

Tabela 6 - Parâmetros das isotermas para o bagaço de cana

\begin{tabular}{|c|c|c|c|c|c|c|c|c|}
\hline Modelo (i) & Tratamento & $\mathrm{q}_{\text {máx }}$ & $\begin{array}{c}\text { Desvio } \\
\text { padrão }\end{array}$ & $\mathrm{K}_{\mathrm{i}}$ & $\begin{array}{c}\text { Desvio } \\
\text { padrão }\end{array}$ & $\mathrm{n}_{\mathrm{i}}$ & $\begin{array}{c}\text { Desvio } \\
\text { padrão }\end{array}$ & $\mathrm{r}^{2}$ \\
\hline \hline \multirow{4}{*}{ Langmuir } & In natura & 10,9712 & 1,0095 & 0,0472 & 0,0139 & - & - & 0,9617 \\
\cline { 2 - 9 } & Ácido & 17,3189 & 1,4818 & 0,0328 & 0,0079 & - & - & 0,9789 \\
\cline { 2 - 9 } & Básico & 129,6238 & 49,5778 & 0,0430 & 0,0204 & - & - & 0,9958 \\
\hline \multirow{4}{*}{ Freundlich } & In natura & - & - & 1,4656 & 0,2843 & 2,6520 & 0,2989 & 0,9709 \\
\cline { 2 - 9 } & Ácido & - & - & 1,5850 & 0,1963 & 2,2267 & 0,1381 & 0,9922 \\
\cline { 2 - 9 } & Básico & - & - & 5,5414 & 0,3479 & 1,1451 & 0,0484 & 0,9973 \\
\hline \multirow{4}{*}{ Tóth } & In natura & 5,9876 & 6,3410 & 0,4811 & 0,4481 & 0,3556 & 0,2536 & 0,9830 \\
\cline { 2 - 9 } & Ácido & 15,1021 & 42,5017 & 0,5581 & 0,4718 & 0,2264 & 0,2324 & 0,9925 \\
\cline { 2 - 9 } & Básico & $1,8292 \mathrm{E}^{7}$ & $2,8811 \mathrm{E}^{10}$ & 0,1410 & 0,4108 & 0,0252 & 3,4365 & 0,9960 \\
\hline \multirow{3}{*}{ Sips- } & In natura & 3,3826 & 2,0314 & 0,2924 & 0,3138 & 0,7187 & 0,0790 & 0,9873 \\
\cline { 2 - 9 } & Ácido & 3,4911 & 2,8536 & 0,3194 & 0,4229 & 0,6223 & 0,0851 & 0,9922 \\
\cline { 2 - 9 } & Básico & 0,1937 & 15,4985 & 46,9090 & $4,2665 \mathrm{E}^{3}$ & 0,1279 & 0,1264 & 0,9970 \\
\hline & In natura & 15,9157 & 6,3969 & 0,0137 & 0,0196 & 0,6241 & 0,1887 & 0,9797 \\
\cline { 2 - 9 } & Ácido & 33,9282 & 23,0316 & 0,0043 & 0,0090 & 0,6003 & 0,1493 & 0,9926 \\
\cline { 2 - 9 } & Básico & $6,4001 \mathrm{E}^{3}$ & $7,0426 \mathrm{E}^{7}$ & $2,2367 \mathrm{E}^{-5}$ & 0,0282 & 0,8736 & 0,2303 & 0,9960 \\
\hline
\end{tabular}

Tabela 7 - Parâmetros das isotermas para a fibra de coco

\begin{tabular}{|c|c|c|c|c|c|c|c|c|}
\hline Modelo (i) & Tratamento & qmáx & $\begin{array}{l}\text { Desvio } \\
\text { padrão }\end{array}$ & $\mathrm{K}_{\mathrm{i}}$ & $\begin{array}{l}\text { Desvio } \\
\text { padrão }\end{array}$ & $\mathrm{n}_{\mathrm{i}}$ & $\begin{array}{l}\text { Desvio } \\
\text { padrão }\end{array}$ & $\mathrm{r}^{2}$ \\
\hline \multirow{3}{*}{ Langmuir } & In natura & $2,9562 \mathrm{E}^{4}$ & $6,0495 \mathrm{E}^{6}$ & $1,1,7234 \mathrm{E}^{-5}$ & $1,3144 \mathrm{E}^{-6}$ & - & - & 0,9592 \\
\hline & Ácido & 41,3602 & 8,1326 & 0,0305 & 0,0118 & - & - & 0,9744 \\
\hline & Básico & $3,1013 \mathrm{E}^{4}$ & $9,3468 \mathrm{E}^{6}$ & $5,1462 \mathrm{E}^{-5}$ & 0,0155 & - & - & 0,9663 \\
\hline \multirow{3}{*}{ Freundlich } & In natura & - & - & 0,1313 & 0,0138 & 0,7248 & 0,0153 & 0,9993 \\
\hline & Ácido & - & - & 1,8207 & 0,5671 & 1,4737 & 0,1912 & 0,9686 \\
\hline & Básico & - & - & 1,2360 & 0,4516 & 0,9107 & 0,1148 & 0,9720 \\
\hline \multirow{3}{*}{ Tóth } & In natura & 266,7928 & $6,2444 \mathrm{E}^{6}$ & 0,0039 & 2,2254 & 0,0055 & 183,3319 & 0,9166 \\
\hline & Ácido & $3,1380 \mathrm{E}^{4}$ & $6,8224 \mathrm{E}^{6}$ & $3,4540 \mathrm{E}^{-5}$ & 0,0075 & 3,2947 & 72,5383 & 0,9661 \\
\hline & Básico & $7,5843 \mathrm{E}^{3}$ & $4,3771 \mathrm{E}^{7}$ & $2,0766 \mathrm{E}^{-4}$ & 1,3255 & 0,0322 & $3,1456 \mathrm{E}^{3}$ & 0,9481 \\
\hline \multirow{3}{*}{$\begin{array}{l}\text { Radke- } \\
\text { Praunsnitz }\end{array}$} & In natura & 0,9889 & 2,8209 & 0,1949 & 0,4517 & $-0,4664$ & 0,1989 & 0,9992 \\
\hline & Ácido & $5,3950 \mathrm{E}^{3}$ & $9,2495 \mathrm{E}^{6}$ & $2,2232 \mathrm{E}^{-4}$ & 0,3814 & 82,8156 & $1,4197 \mathrm{E}^{5}$ & 0,9630 \\
\hline & Básico & 0,0019 & 21,8474 & 364,6839 & $3,8192 \mathrm{E}^{6}$ & $-0,0981$ & 0,4604 & 0,9580 \\
\hline \multirow{3}{*}{ Sips } & In natura & $1,6357 \mathrm{E}^{4}$ & $1,7381 \mathrm{E}^{6}$ & $2,0310 \mathrm{E}^{-4}$ & 0,0248 & 1,3799 & 0,1823 & 0,9990 \\
\hline & Ácido & 26,3011 & 6,6446 & 0,0858 & 0,0546 & 1,6775 & 1,0723 & 0,9666 \\
\hline & Básico & 33,7048 & 20,2744 & 0,1151 & 0,1020 & 2,0702 & 1,5479 & 0,9660 \\
\hline
\end{tabular}

Ao realizar o mesmo estudo para os outros testes, é possível perceber que em todos os casos o modelo de Freundlich foi o que se ajustou melhor. De acordo com Tonucci (2014), para o modelo de Freundlich, $\mathrm{K}_{\mathrm{F}}$ indica a capacidade de adsorção do adsorvente. Dito isso, observou-se que o bagaço de cana com tratamento básico apresentou capacidade de adsorção aproximadamente cinco vezes maior do que as outras variações dos adsorventes, indicando esta fibra com este tratamento, como uma possibilidade de otimizar processos de adsorção em larga escala. Analisando ainda o parâmetro $\mathrm{K}_{\mathrm{F}}$, notou-se que a fibra de coco in natura apresentou o pior desempenho. O bagaço de cana in natura e com tratamento ácido e a fibra de coco com tratamento ácido e básico mostraram resultados semelhantes. 


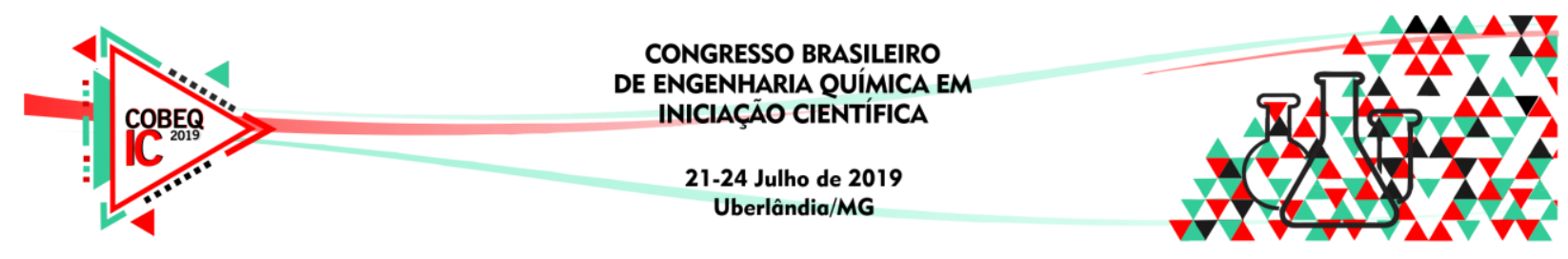

\section{CONCLUSÃO}

Através deste trabalho foi possível concluir que, independentemente da fibra utilizada, o sistema apresentou um comportamento de pseudo-segunda-ordem para todos os testes. Além disso, notou-se que a velocidade inicial de adsorção aumenta proporcionalmente à concentração de adsorvato em solução.

As isotermas de equilíbrio se ajustaram ao mesmo modelo, de Freundlich, uma vez que os outros quatro testados tiveram seus parâmetros com variância elevada, indicando a inconsistência destes. Pela análise da constante de Freundlich foi possível perceber uma significância dos resultados alcançados com o bagaço de cana tratado com solução básica $(\mathrm{NaOH})$, sendo então esta fibra a mais indicada a ser aplicado em larga escala, uma vez que sua capacidade de adsorção foi cerca de 5 vezes maior, quando comparado às outras fibras utilizadas. Isso não descarta o uso dos outros tratamentos, pois estes também apresentaram viabilidade, aumentando a eficiência de adsorção.

\section{REFERÊNCIAS}

ALBINANTE, S. R.; PACHECO, E. B. A. V.; VISCONTE, L. L. Y. Revisão dos tratamentos químicos da fibra natural para mistura com poliolefinas. Química nova, v. 36, n. 1, p. 114-122, 2013.

BERTOLINI, T. C. R.; FUNGARO, D. A. Estudos de Equilíbrio e Modelagem Cinética da Adsorção do Corante Cristal Violeta sobre Zeólitas de Cinzas Leve e Pesada de Carvão. In: $3^{\text {rd }}$ International Workshop: Advances in Cleaner Production. São Paulo, 2011.

COLOMBO, A. Biossorção dos íons cádmio e chumbo pela casca de soja. Tese de mestrado em Engenharia Química, Universidade Estadual do Oeste do Paraná. Toledo, 2013.

FURMANSKI, L. $\quad$ M.; COSTA, P. D.; DOMINGUINI, L. Estudo da cinética de adsorção de azul de metileno por resíduos fibrosos de bananeira. Conference Paper, 2012.

GUILARDUCI, V. V. S. Adsorção de fenol sobre carvão ativado em meio alcalino. Química nova, v. 29, n. 6, p. 1226-1232, 2006.

JORGE, I. R.; TAVARES, F.P.; SANTOS, K. G. Remoção do corante azul de metileno no tratamento de efluentes por adsorção em bagaço de cana de açúcar. XXXVII ENEMP, 2015.

LAVICH, R. R. Simulação de uma coluna de adsorção em leito fixo para a remoção de poluentes de gás e petróleo. Monografia final, Departamento de Engenharia Química, Universidade Federal de Santa Catarina. Florianópolis, 2003.

SILVA, W. L. L.; OLIVEIRA, S. P. Modificação das características de adsorção do bagaço de cana para remoção do azul de metileno de soluções aquosas. Scientia Plena, v. 8, n. 9, 2012.

TONUCCI, M. C. Adsorção de diclofenaco, estradiol e sulfametoxazol em carvões ativados e nanotubos de carbono: estudos cinéticos e termodinâmicos. Tese de mestrado em Engenharia Ambiental, Universidade Federal de Ouro Preto. Ouro Preto, 2014. 